\title{
Efeito do silício na qualidade de flores de Dendrobium nobile (Orchidaceae)
}

\section{Effect of silicon on the quality of flowers of Dendrobium nobile (Orchidaceae)}

\author{
Patrícia Reiners Carvalho ${ }^{1 *}$; Ricardo Tadeu Faria ${ }^{2}$; \\ Inês Cristina de Batista Fonseca ${ }^{2}$; Oscar de Andrade Junior ${ }^{1}$
}

\begin{abstract}
Resumo
A orquídea Dendrobium nobile Lindl é amplamente cultivada pelo mundo como flor de corte e em vasos. O silício ( $\mathrm{Si}$ ) tem demonstrado efeito benéfico em diversas culturas, como por exemplo: aumentando a rigidez celular, conferindo proteção à fitopatógenos, aumento da capacidade fotossintética, tolerância à seca, promoção de maior crescimento e conservação pós-colheita. O objetivo deste trabalho foi avaliar o efeito do silício em diferentes concentrações na qualidade de flores da orquídea Dendrobium nobile. Os tratamentos foram realizados com silicato de magnésio $(\mathrm{SiMg})$ nas seguintes concentrações: 0,$0 ; 0,16$; 0,$32 ; 0,48$ e $0,64 \mathrm{~g} \mathrm{~L}^{-1}$. As aplicações foram realizadas quinzenalmente via foliar, totalizando quatro aplicações dois meses antes do florescimento. As variáveis avaliadas foram: longevidade das flores, número de flores por vaso e número de brotos por planta. O delineamento experimental foi inteiramente casualizado com cinco tratamentos de dez repetições. As aplicações foliares de SiMg incrementaram a produção de flores em média de $59 \%$ e de brotos em até $66 \%$, mostrando-se mais eficiente de acordo o aumento das concentrações testadas. Observa-se o aumento da longevidade das flores na dose $0,45 \mathrm{~g}$ $\mathrm{L}^{-1}$ em até quatro dias.
\end{abstract}

Palavras-chave: Silicato de magnésio, orquídea, nutrição, flor de vaso

\begin{abstract}
The orchid Dendrobium nobile Lindl is widely cultivated throughout the world as cut and potted flowers. Silicon $(\mathrm{Si})$ has demonstrated beneficial effects on various crops, increasing cell stiffness, giving protection to pathogens, increasing photosynthetic capacity and drought tolerance, promoting higher growth and longevity. The aim of this study was to evaluate the effect of different concentrations of silicon on Dendrobium nobile orchid flowers. Treatments were performed with magnesium silicate ( $\mathrm{SiMg}$ ) at the following concentrations: $0.0,0.16 ; 0.32 ; 0.48$ and $0.64 \mathrm{~g} \mathrm{~L}^{-1}$. The foliar applications were carried out fortnightly totaling four applications two months before flowering. The variables evaluated were: longevity of flowers, number of flowers per pot and number of shoots per plant. The experimental design was completely randomized with five treatments and ten replications. The foliar applications of SiMg increased 59\% flower production and shoots up to $66 \%$, being more efficient with the increasing of concentrations. The increasing of longevity of the flowers in the dose $0.45 \mathrm{~g} \mathrm{~L}-1$ was up to four days. Key words: Magnesium silicate, orchid, nutrition, potted flower
\end{abstract}

\footnotetext{
${ }^{1}$ Discente(s) de Doutorado de Agronomia da Universidade Estadual de Londrina, UEL, Londrina, PR. E-mail: patriciareiners@ superig.com.br; oscar@unoeste.br

${ }^{2}$ Profs. Drs. do Dept ${ }^{\circ}$ de Agronomia, UEL, Londrina, PR. E-mail: faria@uel.br; inescbf@uel.br

* Autor para correspondência
} 


\section{Introdução}

No Brasil, a floricultura vem adquirindo notável desenvolvimento ao longo dos últimos anos e, as principais flores comercializadas são: rosas, crisântemos e orquídeas (JUNQUEIRA; PEETZ, 2007). Este setor do agronegócio chega a exportar cerca de US\$ 31 milhões ao ano (JUNQUEIRA; PEETZ, 2010).

O gênero Dendrobium (Orchidaceae) é composto por mais de 1100 espécies amplamente distribuído pelo mundo, vindo desde o sul da Ásia ao leste de Nova Guiné e Austrália (PUCHOOA, 2004; XU et al., 2006). A espécie Dendrobium nobile é uma epífita valorizada pela beleza e colorido de suas flores (NAYAK et al., 2000; LORENZI; SOUZA, 2008), com seu lugar garantido no mercado consumidor. Além do seu uso como planta ornamental, vem sendo também referenciada pela literatura chinesa como planta medicinal (YE; QIN; ZHAO, 2002).

O Si é o segundo elemento químico mais abundante na terra afirma Raven et al. (2003), muitas plantas entre elas as ornamentais são acumuladoras desse elemento como mostram os estudos de Voogt e Sonnenfeld (2001), que observaram absorção significativa em Gerbera jamesonii, Rosa spp e Alstroemeria pelegrina. Frantz et al. (2008) encontraram concentrações de $\mathrm{Si}$ em tecido foliar de quatorze espécies vegetais ornamentais. Mattson e Leatherwood (2010) também avaliaram cerca de vinte quatro espécies de plantas ornamentais e encontraram $\mathrm{Si}$ acumulado em diferentes dosagens de acordo com cada espécie estudada.

Apesar de não ser considerado elemento essencial às plantas, o Si tem demonstrado efeito benéfico em diversas culturas aumentando a rigidez celular, conferindo proteção às plantas, aumento da capacidade fotossintética, tolerância à seca, Epstein (2001), além de propiciar melhor conservação póscolheita, como por exemplo, da alface (RESENDE et. al., 2005). No cultivo de orquídeas são escassos os trabalhos com a utilização de Si (VENDRAME et. al., 2010).
O objetivo deste trabalho foi avaliar o efeito do $\mathrm{Si}$, aplicado via foliar em diferentes concentrações, na qualidade de flores da orquídea Dendrobium nobile.

\section{Material e Métodos}

Este experimento foi instalado no Centro de Ciências Agrárias da Universidade Estadual de Londrina (UEL), Estado do Paraná, Brasil. O viveiro utilizado para a realização do experimento foi protegido com tela de polipropileno de coloração preta, com retenção de $50 \%$ do fluxo de radiação solar. A temperatura média durante o período do experimento foi de $25 \pm 8^{\circ} \mathrm{C}$ com umidade relativa de 50 a $60 \%$, clima subtropical (CFA).

Foram selecionadas 50 plantas de Dendrobium nobile com o mesmo padrão de porte e idade, as quais foram obtidas de sementes germinadas in vitro, no Laboratório de Cultura de Tecidos Vegetal da Universidade Estadual de Londrina, com idade média de três anos, com altura média de $25,0 \mathrm{~cm}$, plantadas em vaso preto de polipropileno número dois, com 15,0 cm de altura e 12,5 cm de diâmetro. As irrigações foram realizadas manualmente duas vezes por semana e as adubações aplicadas a cada 15 dias, via foliar, utilizando o fertilizante comercial com Biofert Plus ${ }^{\circledR}$, que contém $8 \%$ de nitrogênio, $9 \%$ de fósforo, $9 \%$ de potássio, $1 \%$ de cloro, $1 \%$ de enxofre, $0,15 \%$ de ferro, $0,2 \%$ de cobre, $035 \%$ de zinco, $0,02 \%$ de boro, $0,2 \%$ de manganês, $0,6 \%$ de magnésio, $0,005 \%$ de cobalto e $0,005 \%$ de molibdênio, na concentração de $5,0 \mathrm{ml} \mathrm{L}^{-1}$, o substrato dos vasos sendo composto por carvão vegetal + casca de pinus compostado $(1: 1 \mathrm{v} / \mathrm{v})$ (DRONK et al., 2012).

O delineamento experimental utilizado foi o inteiramente casualizado, com cinco tratamentos e dez repetições e um vaso com uma planta por parcela. Os tratamentos consistiram de aplicações com silicato de magnésio ( $\mathrm{SiMg}$ ), composto de $65 \%$ de $\mathrm{SiO}_{2}$ e $14 \%$ de $\mathrm{MgO}$, pulverizadas manualmente com $0,004 \mathrm{~L}$ de cada solução por planta, nas 
seguintes concentrações: 0,0 (controle); 0,16 ; 0,32; 0,48 e $0,64 \mathrm{~g} \mathrm{~L}^{-1}$, sendo realizada quinzenalmente via foliar, totalizando quatro aplicações dois meses antes do florescimento.

As variáveis analisadas durante os meses de junho, julho, agosto e setembro de 2010 foram: longevidade das flores, número de flores por planta e número de brotos por planta. Para as avaliações as plantas permaneceram em seus respectivos vasos e durante o florescimento foram transferidas para uma sala e ficaram sobre uma bancada, com oscilação da temperatura entre 21 e $22^{\circ} \mathrm{C}$ e umidade relativa de 50 a $60 \%$. As avaliações foram realizadas a cada dois dias até o término do florescimento, acompanhandose o critério de notas, seguindo o modelo de Assis et al. (2003), mostrado na Figura 1.

Figura 1. Critério de notas utilizadas para avaliação da qualidade estética das flores de Dendrobium nobile.

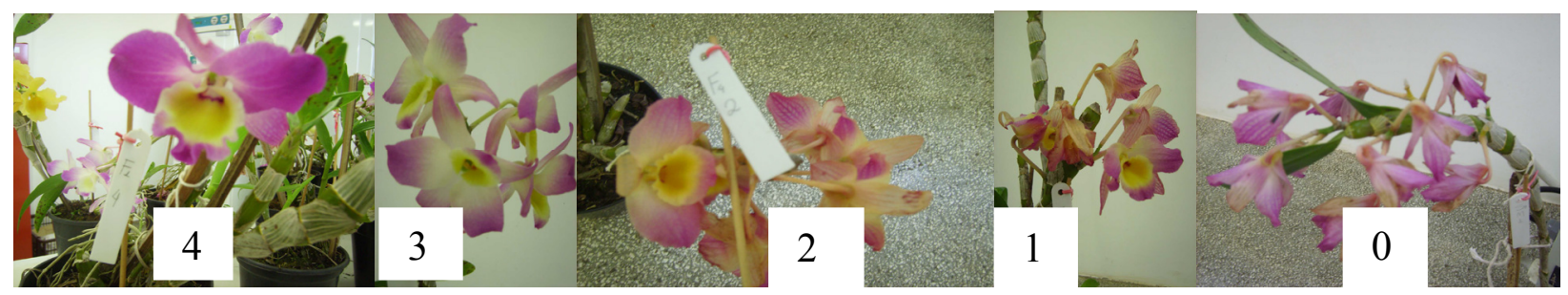

Nota 4 - flor com características perfeitas para comercialização, túrgida, vistosa e sem manchas.

Nota 3 - flor com alguma destas características alterada, mas ainda com qualidade comercial.

Nota 2 - flor com pétalas e sépalas levemente murchas e ou manchadas, não adequada para a comercialização.

Nota 1 - flor com pétalas e sépalas murchas e manchadas, não adequada para a comercialização.

Nota 0 - flor com pétalas e sépalas ressecadas ou sem estas.

Fonte: Elaboração dos autores.

Notas inferiores a três representam flores sem valor comercial. Como, entretanto as hastes possuem flores em vários estágios de maturação (de botões a flores abertas), no presente trabalho foram consideradas apenas as flores que estavam desabrochando no início da avaliação.

Os dados foram submetidos à ANOVA com teste $\mathrm{F}$ ao nível de 5\% de probabilidade e ajustadas equações de regressão utilizando o programa “SISVAR" versão 4.2 (FERREIRA 2003).

As notas médias obtidas foram submetidas à análise de regressão nas diferentes concentrações de SiMg aplicado nas plantas de Dendrobium nobile aos vinte e dois dias de seu florescimento. Este dia foi escolhido pois nessa ocasião foi encontrado um único tratamento com plantas com nota 3 , ou seja, com condições de comercialização, diferenciandose dos outros que apresentavam notas inferiores a 3, ou seja, sem condições de comercialização.
Posteriormente, através da equação da derivada primeira, foi calculado o ponto de máximo na dose.

\section{Resultados e Discussão}

As médias das notas obtidas para longevidade das flores sob os diferentes tratamentos são mostrados na Tabela 1. Observa-se que do primeiro ao décimo primeiro dia do florescimento, não houve diferença significativa entre os tratamentos, apresentando todas as notas próximas ou iguais a quatro.

Na Tabela 1, verifica-se que a partir do vigésimo dia do florescimento tem-se efeito benéfico do uso do SiMg nas dosagens de 0,48 e $0,64 \mathrm{~g}$. $\mathrm{L}^{-1}$, pois as plantas ainda tinham condições de comercialização enquanto os outros tratamentos já se encontravam com notas abaixo de 3, ou seja, flor com pétalas e sépalas levemente murchas e ou manchadas, não adequada para a comercialização. 
Tabela 1. Notas atribuídas às flores de Dendrobium nobile submetidas a diferentes concentrações de SiMg.

\begin{tabular}{|c|c|c|c|c|c|c|c|c|c|}
\hline \multirow{2}{*}{ Tratamentos } & \multicolumn{9}{|c|}{ Dias após o início do florescimento } \\
\hline & $1 .^{\circ}$ & $12 .^{\circ}$ & $14 .^{\circ}$ & $16^{\circ}$ & $18 .^{\circ}$ & $20 .^{\circ}$ & $22 .^{\circ}$ & $24 .^{\circ}$ & $26^{\circ}$ \\
\hline & \multicolumn{9}{|c|}{ Notas } \\
\hline 0,0 controle & $4^{1}$ & 3,50 & 3,45 & 3,32 & 3,05 & 2,55 & 1,87 & 1,12 & 0,70 \\
\hline 0,16 SiMg L-1 & 4 & 3,44 & 3,42 & 3,30 & 3,10 & 2,62 & 2,62 & 1,54 & 0,72 \\
\hline $0,32 \mathrm{SiMg} \mathrm{L}^{1}$ & 4 & 3,65 & $3,62 \mathrm{ab}$ & 3,62 & 3,54 & 2,98 & 2,98 & 1,66 & 0,83 \\
\hline 0,48 SiMg L-1 & 4 & 3,69 & $3,66 \mathrm{a}$ & 3,66 & 3,60 & 3,20 & 3,20 & 2,33 & 0,89 \\
\hline $0,64 \mathrm{SiMg} \mathrm{L}^{1}$ & 4 & 3,60 & $3,55 \mathrm{ab}$ & 3,55 & 3,48 & 3,12 & 2,90 & 1,77 & 0,68 \\
\hline $\mathrm{CV} \%$ & 0 & 9,15 & 6,54 & 8,52 & 8,65 & 10,64 & 9,75 & 21,33 & 18,96 \\
\hline Médias & 4 & 3,57 & 3,54 & 3,49 & 3,35 & 2,89 & 2,71 & 1,68 & 0,58 \\
\hline
\end{tabular}

Fonte: Elaboração dos autores.

No vigésimo segundo dia do florescimento apenas a dose de $0,48 \mathrm{~g} \mathrm{~L}^{-1}$ apresentava flores com nota 3 , estando todos os outros tratamentos com sinais de senescência e perda de qualidade das flores, demonstrando assim o aumento da longevidade da flor em até quatro dias em relação ao controle de orquídea Dendrobium nobile com aplicação de $\mathrm{SiMg}$ nesta dosagem, destacado na Figura2. Fazendo a derivada primeira da equação mostrada no gráfico da Figura 2, encontra-se o ponto de máximo na dose de $0,45 \mathrm{~g} \mathrm{~L}^{-1}$.

Figura 2. Notas de longevidade atribuídas às flores de Dendrobium nobile aos vinte e dois dias de seu florescimento em função das concentrações de SiMg.

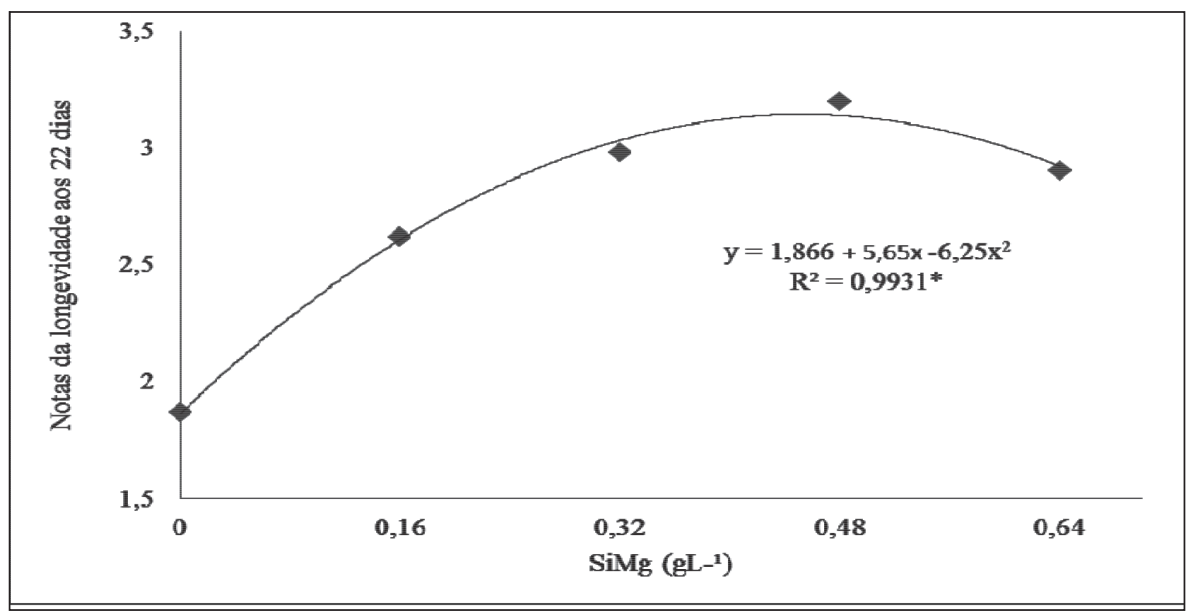

Fonte: Elaboração dos autores.

Epstein (1999) e Marschner (1986) afirmam que o Si reduz o estresse hídrico por propiciar menor transpiração das plantas. Diminuindo a taxa de transpiração da planta, ela por conseqüência gasta menos açúcares para sua manutenção metabólica. Çelikel e Reid (2002) relata que a manutenção da qualidade floral ocorre em função do fornecimento de açúcares exógenos que mantém o volume da matéria seca das flores e o nível de substratos respiratórios, especialmente nas pétalas, conservando a respiração vital e prolongando a longevidade. Sendo assim, se as pétalas continuarem recebendo açúcares e sua respiração for mantida por mais tempo, maior será sua longevidade. Além disso, o Si regulando a 
transpiração, a possibilidade de necrose dos tecidos e o aparecimento de manchas de senescência por falta de água é menor.

Na Figura 3 observa-se o aumento do número de flores e brotos por planta conforme aumenta a concentração de SiMg. O aumento do número de flores também foi observado em rosas, quando o $\mathrm{Si}$ foi acrescentado em solução nutritiva em 50 e 100 ppm (REZZI; BABALAR; KALANTARI, 2009).

Os resultados apresentados neste estudo (Figura 3) mostram que houve um incremento na produção de flores e brotos nas orquídeas com os tratamentos de SiMg. Estudos têm demonstrado que o uso de Si é benéfico para o crescimento de plantas, principalmente em monocotiledôneas e alivia os estresses bióticos e abióticos em plantas (EPSTEIN, 1994; VOOGT; SONNEVELD, 2001), permitindo que estes dois fatores associados melhorem $\mathrm{o}$ desempenho das plantas tratadas com SiMg.

Para produção em estufa, a maioria das plantas é cultivada em recipientes usando substratos sem solo como meio de crescimento, o que limita a disponibilidade do Si para as plantas em comparação com solos minerais (VOOGT; SONNEVELD, 2001). Nesse caso, o fornecimento via foliar do Si, como no presente trabalho, é interessante por seus efeitos positivos no crescimento e desenvolvimento de plantas de Dendrobim nobile.

Figura 3. Número de flores (A) e número de brotos (B) em plantas de Dendrobim nobile em função das concentrações de SiMg.

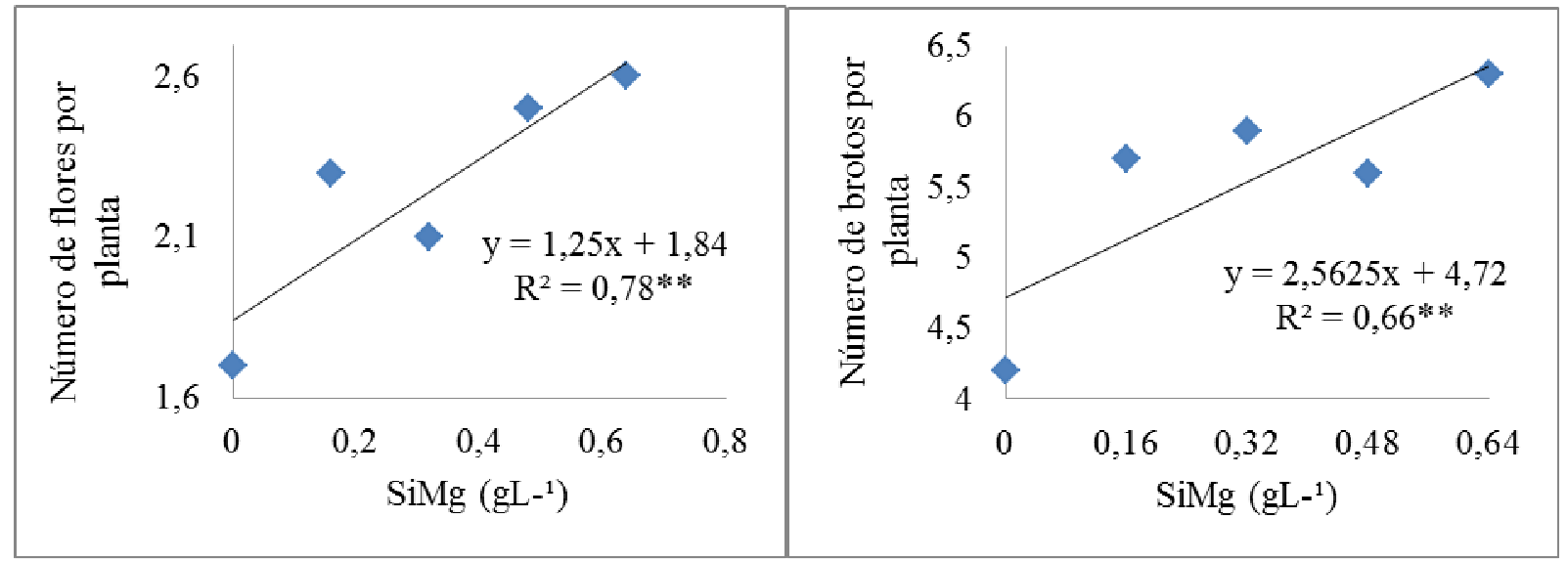

Fonte: Elaboração dos autores.

Os efeitos positivos observados na maior produção de flores e brotos (Figura 3) em função da maior disponibilidade de Si para as plantas de Dendrobium nobile também foram relatados em estudo realizado por Muir et al. (1999), os quais constataram um maior número de brotações $(25 \%)$ e de flores (62\%), em comparação com o tratamento controle em margaridas (Helichrysum adenohorum).

O produto utilizado, $\mathrm{SiMg}$, além do silício também fornece o macronutriente magnésio, o qual pode ter interferido nos resultados obtidos, já que é componente da clorofila e por isso importante na fotossíntese. Além disto, o magnésio estimula a formação de açúcares, proteínas, gorduras e vitaminas vegetais, além de influenciar positivamente o engrossamento das paredes e permeabilidade das membranas celulares (TAIZ; ZEIGER, 2009). 


\section{Conclusões}

O fornecimento de $0,45 \mathrm{~g} \mathrm{~L}^{-1}$ de SiMg promove o aumento da longevidade de flores de Dendrobium nobile em quatro dias.

Incrementos nas concentrações de $\mathrm{SiMg}$ aumentam o número de flores e brotos de plantas de Dendrobium nobile.

\section{Referências}

ASSIS, A. M.; COLOMBO, L. A.; FARIA, R. T.; FONSECA, I. C. B. Longevidade pós-colheita de pseudobulbos com flores de Dendrobium nobile (Orchidaceae). Revista Brasileira de Horticultura Ornamental, Campinas, v. 9, n. 1, p. 85-87, 2003.

ÇELIKEL, F. G.; REID, M. S. Storage temperature affects the quality of cut flowers from the Asteraceae. HortScience, Alexandria, v. 37, n. 1, p. 148-150, 2002.

DRONK, A. G.; SILVA, A. P. V.; CUQUEL, F. L.; FARIA, R. T. Desenvolvimento vegetativo de híbrido de orquídea em diferentes substratos. Semina: Ciências Agrárias, Londrina, v. 33, n. 6, p. 2109-2114, 2012.

EPSTEIN, E. Silicon in plants: facts vs. concepts. In: DATNOFF, L. E.; SNYDER, G. H.; KORNDÖRFER, G. H. (Ed.). Silicon in agriculture. Amsterdam: Elsevier, 2001. p. 1-15.

. Silicon. Annual Review of Plant Physiology and Plant Molecular Biology, Palo Alto, n. 50, p. 641-664, 1999.

The anomaly of silicon in plant biology. Proceeding of the National Academy of Sciences, USA, v. 91, n. 1, p. 11-17, 1994.

FERREIRA, D. F. Sisvar versão 4.2. Lavras: DEX/ UFLA, 2003.

FRANTZ, J. M.; LOCKE, J. C.; DATNOFF, L.; OMER, M.; WIDRIG, A.; STURTZ, D.; HORST, L.; KRAUSE, C. R. Detection, distribution, and quantification of silicon in floricultural crops utilizing three distinct analytical methods. Communications in Soil Science and Plant Analysis, USA, v. 39, n. 17-18, p. 2734-2751, 2008.

JUNQUEIRA, A. H.; PEETZ, M. S. Análise conjuntural do comércio exterior da floricultura brasileira: balanço 2009 e perspectivas 2010. 2010. Disponível em: $<\mathrm{http}: / /$ www.hortica.com.br/publica.php>. Acesso em: 12 jun. 2011.
Producción y comercialización de plantas ornamentales en Brasil. Horticultura Internacional, Tarragona, Espanha, ano XIV, n. 55, p. 16-19, 2007.

LORENZI, H.; SOUZA, H. M. Plantas ornamentais no Brasil: arbustivas, herbáceas e trepadeiras. 4. ed. Nova Odessa: Plantarum, 2008.

MARSCHNER, H. Mineral nutrition of higher plants. London: Academic Press, 1986.

MATTSON, N. S.; LEATHERWOOD, W. R. Potassium silicate drenches increase leaf silicon content and affect morphological traits of several floriculture crops grown in a peat-based substrate. HortScience, Alexandria, v. 45, n. 1, p. 43-47, 2010.

MUIR, S. C.; KHOO, B. K.; MCCABE, G.; FENSOM, C.; OFFORD, J.; BRIEN, B. S. Some effects of silicon in potting mixes on growth and protection of plants against fungal diseases. In: DATNOFL, E. F.; SNYDER, G. H.; KORNDORFER, G. H. (Ed.). Silicon in agriculture. Amsterdam, The Netherlands: Elsevier, 1999. p. 374. (Studies in plant sciences, 8).

NAYAK, N. R.; SAHOO, S.; PATNAIK, S.; RATH, S. P. Establishment of thin cross section (TCS) culture method for rapid micropropagation of Cymbidium aloifolium (L.) Sw. and Dendrobium nobile Lindl. (Orchidaceae). Scientific Horticulture, Amsterdam, v. 94, n. 1-2, p. 107116, 2000.

PUCHOOA, D. Comparison of different culture media for the in vitro culture of Dendrobium (Orchidaceae). International Journal of Agriculture e Biology, Pakistan, v. 6, n. 5, p. 884-888, 2004.

RAVEN, J. A. Cycling silicon the role of accumulation in plants. New Phytologist, Oxford, v. 158, p. 419-421, 2003.

REEZI, S.; BABALAR, M.; KALANTARI, S. Silicon alleviates salt stress, decreases malondialdehyde content and affects petal color of saltstressed cut rose (Rosa hybrid L.) "Hot Lady". African Journal of Biotechnology, Nairobi, v. 8, n. 8, p. 1502-1508, 2009.

RESENDE, G. M.; YURI, J. E.; MOTA, J. H.; RODRIGUES JÚNIOR, J. C.; SOUZA, R. J.; CARVALHO, J.G. Produção de alface americana em função de doses e épocas de aplicação de Supa Potássio ${ }^{\circledR}$. Horticultura Brasileira, Brasília, v. 23, n. 2, p. 174-178, 2005.

TAIZ, L.; ZEIGER, E. Fisiologia vegetal. 4. ed. Porto Alegre: Artmed, 2009. 820 p. 
VENDRAME, W. A.; PALMATEER, A. J.; PINARES, A.; MOORE, K. A.; DATNOFF, L. E. Silicon Fertilization affects growth of hybrid Phalaenopsis orchid liners. Horttechnology, Alexandria, v. 20, n. 3, p. 603-607, 2010.

VOOGT, W.; SONNENFELD, C. Silicon in horticultural crops grown in soilless culture. In silicon in agriculture: studies. In: DATNOFF, L. E.; SNYDER, G. H.; KORNDORFER, G. H. (Ed.). Plant science. The Netherlands: Elsevier Science, 2001. p. 115-131.
XU, H.; WANG, Z. T.; DING, X. Y.; ZHOU, K. Y.; $\mathrm{XU}, \mathrm{L}$. S. Differentiation of Dendrobium species used as "Huangcao Shihu" by rDNA ITS sequence analysis. Planta Medica, Stuttgart, v. 72, n. 1, p. 89-92, 2006.

YE, Q.; QIN, G.; ZHAO, W. Immunomodulatory sesquiterpene glycosides from Dendrobium nobile. Phytochemistry, Shanghai, v. 61, n. 8, p. 885-890, 2002. 
